\title{
COMPUTATION OF ELECTRO-MAGNETIC TRANSIENTS USING DUAL OR MULTIPLE TIME STEPS
}

\author{
Adam Semlyen Francisco de León ${ }^{*}$ \\ Department of Electrical Engineering \\ University of Toronto \\ Toronto, Ontario, Canada, M5S 1A4 \\ * On leave from Instituto Politecnico Nacional, Mexico
}

\begin{abstract}
When computing transients with the EMTP (the Electro-Magnetic Transients Program) a single time step is selected for the trapezoidal integration all over the system. When this step is small because of the presence of a "fast" component, the simulation may be significantly slowed down. This paper presents a procedure by which the computations at selected fast buses can be performed with smaller time steps than at the rest of the system. The linkage of the variables at two different time steps is achieved in a simple way at the line-bus interface by a small addition to any existing implementation of an electro-magnetic transients program. Significant computational savings can be obtained without any loss of accuracy.
\end{abstract}

Keywords: Dual time step, Trapezoidal integration, Electromagnetic transients, Stiff systems.

\section{INTRODUCTION}

The computation of electro-magnetic transients via the EMTP requires the selection of a step size $H$ to be used for the entire system. More general purpose simulation programs (such as SPICE [1] and ECA [2]) use a variable steps size integration procedure to accommodate the need of simulating in detail short duration fast transients due to the presence of fast components in the system. The EMTP has no analogous capability for handling stiff systems, because of the serious difficulties that such an approach would involve, and it is not the purpose of this paper to address this problem. Quite often, however, a power system has both slow and fast components, meaning that larger or smaller integration steps are appropriate or required for their modeling. Therefore, with a single, global, small integration step, imposed by the fastest component, the simulation is excessively slowed down and becomes overly expensive. The purpose of this paper is to provide a remedy to this situation by permitting the use of two or several time steps, according to the nature (fast or slow) of the corresponding system components. Thus we will have dual or multiple time steps which remain constant, in contrast with the variable time step approach mentioned above.

Both transmission lines and buses (i.e., the components connected to buses) can be characterized as slow or fast. In the case of transmission lines which are represented with frequency dependent parameters the required time step is closely related to the length of the line expressed by the travel time $\tau$ (at light velocity). Thus, for a $300 \mathrm{~km}$ line we have $\tau=1 \mathrm{~ms}$. To properly

92 SM 595-9 PWRS A paper recommended and approved by the IEEE Power System Engineering Committee of the IEEE Power Engineering Society for presentation at the IEEE/PES 1992 Summer Meeting, Seattle, WA, July 12-16, 1992. Manuscript submitted January 31, 1992; made available for printing May 22, 1992. represent the wave distorsion related to frequency dependence, a time step of $1 / 20$ to $1 / 100$ of $\tau$ is adequate. This is related to the slope of the front of the incident wave resulting from a step or impulse input of the least attenuated line mode in a three phase line, excitations that contain even the highest frequencies. Accurate modeling of shorter lines will require smaller step sizes. The modeling of bus structures will result in very small steps $(\tau=0.1 \mu$ s corresponds to $30 \mathrm{~m})$. Lumped parameter components also have a wide range of time constants. The more detailed the model, the smaller are some of the resultant time constants. For example, in the case of transformer models with detailed representation of internal capacitances [3], [4] and of eddy current effects in the iron and in the windings [5], many time constants become much less than $0.1 \mu \mathrm{s}$. It is thus clear, that the computational efficiency can be significantly increased if the detailed simulation is confined to the fast buses only.

The idea of different time steps for different parts of a dynamic system appears to be attractive even for the general case when the system is modeled by state equations. It is however difficult in that case to characterize some components as being either slow or fast, since normally all dynamic modes, both slow and fast, are present in all state variables. Only in particular cases would the state variables of certain components contain exclusively, or dominantly, only slow or fast modes. If it happens, the terminal variables of a slow component (i.e., the input and output by which it is interfaced with a fast component) may still include fast dynamics. Because of this, no definite attempt has been made in this paper to generalize the dual step size approach (intended for the calculation of transients in systems containing transmission lines) to general dynamic systems or networks. We have, however, outlined the relevant ideas in the Appendix.

The remark made above regarding the existence of components that are clearly and definitely slow in comparison to individual components which include fast dynamics as well, is fundamental and applies also to power systems with transmission lines. If the lines are modeled as lossless, the fast transients (from "fast" buses) are transmitted to the other buses which, otherwise, would have been "slow" according to the dynamics of the local components alone. However, with frequency dependent modeling of the transmission lines - which, of course, is the only appropriate way to represent the physical reality - the fast transients remain practically localized at the fast buses (since they are strongly attenuated over relatively short distances of propagation). Therefore, the use of dual (or multiple) time steps requires, and is developed below in the assumption of, frequency dependent transmission line modeling.

\section{THE DUAL TIME STEP METHODOLOGY}

\subsection{Review of EMTP Procedures}

The approach of using two or more time steps for EMTP calculations requires the identification in the existing program of a convenient location where an interface module between a slow and fast component can be conveniently inserted. For this reason, it is 
convenient to review in very simple terms the way the calculation regarding the propagation of traveling waves and their interaction with components connected at terminal buses is performed in the EMTP. This will also permit us to introduce a few basic notations.

Figure 1 shows the interface of a line with a bus. The following two equations apply:

$$
\begin{gathered}
i_{\text {incident }}+i_{\text {reflected }}=i \\
v_{\text {incident }}+v_{\text {reffected }}=v
\end{gathered}
$$

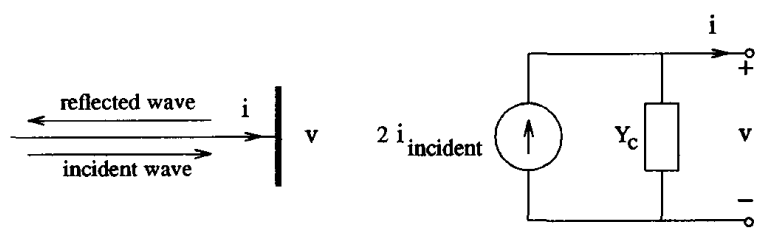

(a)

(b)

Figure 1. Line-bus interface

They are written for instantaneous values of currents and voltages and for their respective components which correspond to the incident and reflected waves in relation to a given bus. They simply express the fact that the resultant values of current and voltage are obtained by the superposition of their wave components. In the case of several conductors, the variables can be considered to be vectors.

If we premultiply $\left(1^{\prime}\right)$ by the characteristic admittance (matrix) $Y_{C}$, we obtain

$$
i_{\text {incident }}-i_{\text {reflected }}=Y_{C} v
$$

Equations (1) and (2) permit to eliminate $i_{\text {reflected }}$. We get

$$
i+Y_{C^{\nu}}=2 i_{\text {incident }}
$$

which can be interpreted as a Norton equivalent (see Figure $1 \mathrm{~b}$ ) for the line end of Figure 1a.

We make at this time the following important remarks:

- In the Norton equivalent (3) (Figure $1 b$ ) $i_{\text {incident }}$ is a known input, equal, in the lossless case, to $-i_{\text {reflected }}$ from the far end at time $\tau$ in the past. In the case of lossy, frequency dependent line modeling, the corresponding relation is, in the frequency domain, $I_{\text {incident }}=-G_{P} I_{\text {reflected }}$ where $G_{P}$ is the propagation transfer function. The time domain equivalent of this latter equation is a convolution which has been implemented in the EMTP in the form of recursive convolutions [6], [7]. This problem is a particular case of a (matrix) transfer function relation of the general form

$$
Y=G U
$$

We use the notation $G$ for a transfer function (matrix) instead of the more usual $H$, because we have reserved the latter to designate the time step.

If (4) is fitted to a set of linear state equations

$$
\begin{aligned}
& \dot{x}=A x+B u \\
& y=C x+D u
\end{aligned}
$$

whose frequency domain realization $C(j \omega-A)^{-1} B+D$ closely approximates $G$ of (4), then trapezoidal discretization of (5) yields the time domain relation (with a different $G$ ):

$$
y=G u+y_{h i s t}
$$

The conclusion for this short discussion is that, whenever frequency dependence is an issue, the relevant frequency domain relation (4) translates to the almost identical (and just as simple) time domain equation (6). The latter contains a history term which reflects the system dynamics.

- The previous remarks apply to both the propagation transfer function $G_{P}$ and the characteristic admittance $Y_{C}$ (or $G_{Y}$ ) for lines with frequency dependent parameters. Therefore, if the history term is added to the Norton source current, equation (3) remains valid for a general line model.

- The state equations (5) and the resulting linear relation (6) are also valid for the representation of the components connected to the bus. If $u$ is voltage and $y$ is current, then we have a Norton representation of the load. Combining the Norton equivalents of all lines connected to a bus (or buses) with that of the load, we solve for the voltage and then for the current of each connected line. Then from (1),

$$
i_{\text {reflected }}=i-i_{\text {incident }}
$$

This information has to be stored as it is the basis for determining the incident wave currents $i_{\text {incident }}$ at the other end of the line, at some later time.

The conclusions of the above discussions are the following:

(a) The input to the bus, i.e., to the Norton equivalent (3), is $i_{\text {incident }}$. It is the output available from the transmission line and is processed together with the components connected to the bus.

(b) The output from the bus and input to the line is $i_{\text {reflected }}$ of (7). Thus the interface variables between the line and the bus are

$$
i_{\text {incident }} \text { and } i_{\text {reflected }}
$$

Therefore, if a dual time step interface is to be implemented between a line and a bus, the problem to be solved is how to reconcile the different sampling rates for the variables (8) at the line side and the bus side of the interface.

(c) The interface variables (8) can be used irrespective of the type of modeling, whether it is frequency dependent or not (see the negative remarks regarding lossless modeling at the end of the Introduction) or whether modes or direct phase domain modeling is used. The only requirement is to insert in the existing program for calculation of transients an interface for time step conversion. Details of this procedure are given in the next section.

\subsection{Two Time Step Interface}

We shall denote the step size on the line side by $H$ and on the bus side by $h$. We shall always assume that

$$
H=n h
$$

where $n$ is a positive integer, larger than unity, except in the trivial case $(H=h)$ when there is no time step conversion. We shall examine below the case when $H>h$.

\subsubsection{Interpolation for Input}

The input $i_{\text {incident }}$ to the Norton equivalent (3) is available from the line side of the interface only at time intervals $H$. Since it is an output from the slow part of the system, its variation over that time interval is very close to linear. Consequently, the values of $i_{\text {incident }}$ to be used as inputs to the bus side of the interface are obtained by linear interpolation between two consecutive values of $i_{\text {incident }}$ from the line side.

\subsubsection{Smoothing for Output}

The output from the bus to the line is $i_{\text {reffected. }}$. It is calculated within the line side step $H$ in $n$ (small) time intervals $h$. Since these results are produced by a "fast" components, $i_{\text {reflected }}$ calculated at the bus side may be oscillatory. Then, the particular single value at the end of the step $H$, needed to be transmitted to the other end of the line, may be (and is in fact quite often, as the computational experience has shown) of an ill-defined (quasi- 
random) value and thus inadequate for reliable calculations. The problem has been solved by smoothing the bus side values $i_{\text {reflected }}$ to an equivalent straight line segment from $i_{\text {reflected }}$ to $i_{\text {reflected }}$. This is done by equating the area under the curve described by the calculated $i_{\text {reffected }}$ (obtained basically by adding up the values of $i_{\text {refiected }}$ ) to the trapezoidal area

$$
\frac{i_{\text {reflected }_{0}}+i_{\text {reflected }}}{2} H
$$

Here $i_{\text {reflected }}$ is known from the previous step, so that $i_{\text {reflected }}$ results directly. It represents the smoothed output to be used at the end of the time interval $H$ for line side calculations.

\subsection{Multiple Time Step Conditions}

As indicated in equation (9), valid where any two time steps are interfaced, the bus side step size $h$ must not be larger than the line side step size $H$,

$$
h \leq H
$$

Since the lines in a system can be of very different lengths and since the shorter ones require smaller time steps for their modeling, it is conceivable that the selection of more than two time steps is useful for the system. Then, according to (11), the bus side time step can be at most equal to that of the shortest (i.e., having the smallest time step) connected line(s). In this way, buses and lines are associated to each other by having the same time constants and subsystems with distinct time constants are formed each with its own time constant.

Figure 2 gives an example of a system with three time step related subdivisions: there exists a slow (S) and a "medium" (M) part and also a single bus that is fast (F), presumably because of a component modeled in great detail.

The methodology described in this paper is applicable to multi-conductor transmission lines by characterizing the line as fast, medium, or slow, in accordance with its least attenuated mode.

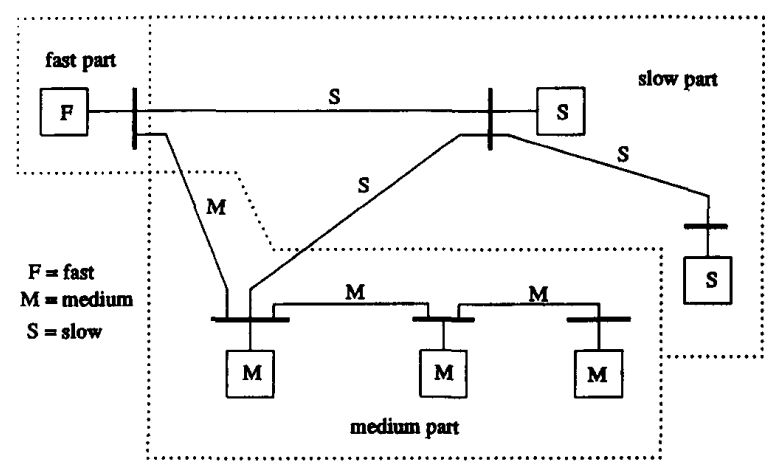

Figure 2. Multiple time step system

\section{SIMULATION RESULTS}

The purpose of the simulations to be described is to validate the feasibility of using dual time steps and to demonstrate both its accuracy and the computational savings that can reasonably be expected. Implementation of the method in the EMTP and its application to computations with complex, three phase components, such as advanced transformer models, is not within the scope of this study.

\subsection{Validation With Small System}

A small system consisting of three buses and three transmission lines (shown in Figure 3) was used as a first example for the validation of the methodology presented in this paper. It contains a "fast" load, a "slow" load, and a source. All transmission lines should be considered "slow" (compared with the fast load) since their lengths are in the hundreds of kilometers. At $t=0$ the system is energized by applying a voltage of $1 \mathrm{~V}$ (or 1 p.u.) at the source bus. The fast transients are excited by giving an initial charge to the capacitor of the fast load.

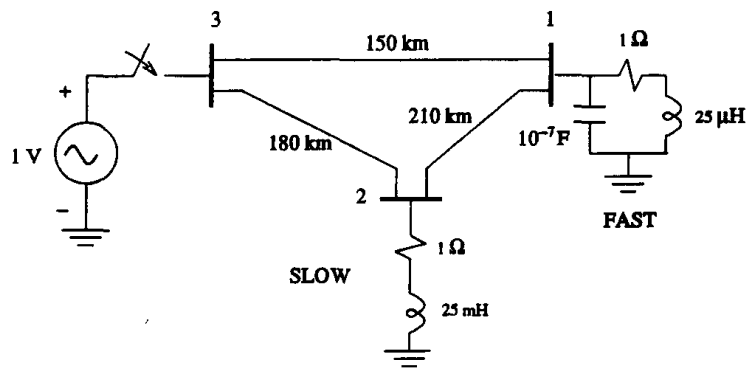

Figure 3. Small system

Figures $4 \mathrm{a}$ and $4 \mathrm{~b}$ show the voltage at buses 1 (fast) and 2 (slow) when the transmission lines are modeled as lossless (ideal). The integration time step used in the simulation was $0.1 \mu \mathrm{s}$ for the entire system (standard procedure). From the figure we can see fast transient oscillations appearing at the slow bus at $0.6 \mathrm{~ms}$ (travel time from the fast bus to the slow bus) and at the fast bus at $1.0 \mathrm{~ms}$ (twice the travel time from the fast bus to the source bus). These high frequency oscillations should be viewed as nonphysical since in the more realistic condition of lossy transmission lines, to be shown next, they are damped out during their propagation (compare Figure 4a with Figure 5a). With a larger integration step these non-physical oscillations are amplified.
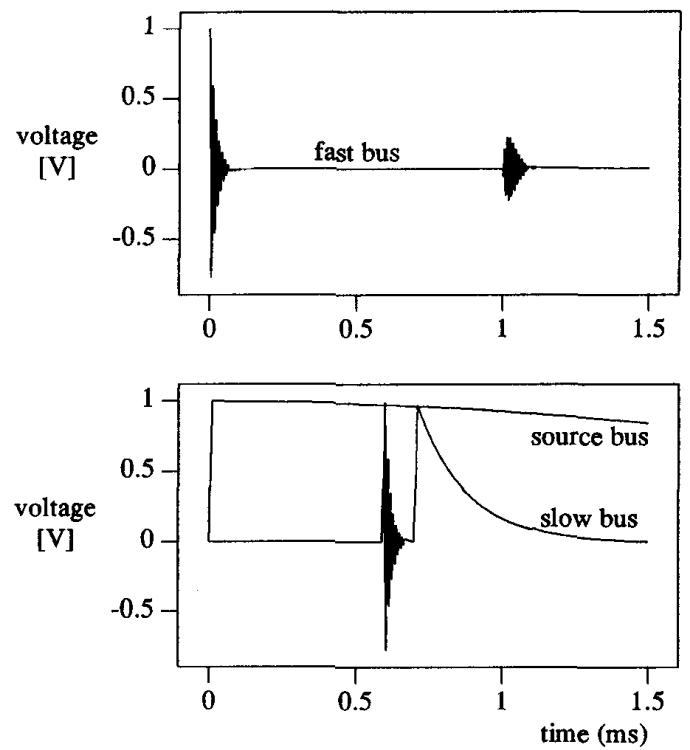

(b)

Figure 4. Voltage at the buses using ideal transmission lines

In conclusion, lossless line modeling in the presence of fast transients may be inappropriate even if a sufficiently small time step is used. Because of this, we examine the adequacy and merits of the dual time steps approach in the case of frequency dependent, lossy line modeling. 
We have considered three methods when the transmission lines are modeled as frequency dependent, namely:

(1) Small, single time step for the entire system, $\mathrm{h}=0.1 \mu \mathrm{s}$. This is the standard procedure in EMTP. Figure 5a shows the voltage at the fast bus. The cpu time on a MIPS-RISC 6280 computer is 9.73 seconds.

(2) Dual time steps, $\mathrm{H}=10 \mu \mathrm{s}$ for the slow part and $\mathrm{h}=0.1 \mu \mathrm{s}$ for the fast part. The voltage at the fast bus for this case is shown in Figure 5b. Cpu time $2.15 \mathrm{~s}$.

(3) Large single time step for the entire system, $\mathrm{H}=10 \mu \mathrm{s}$. In Figure $5 \mathrm{c}$ we show the voltage at the fast bus. Cpu time $0.14 \mathrm{~s}$.

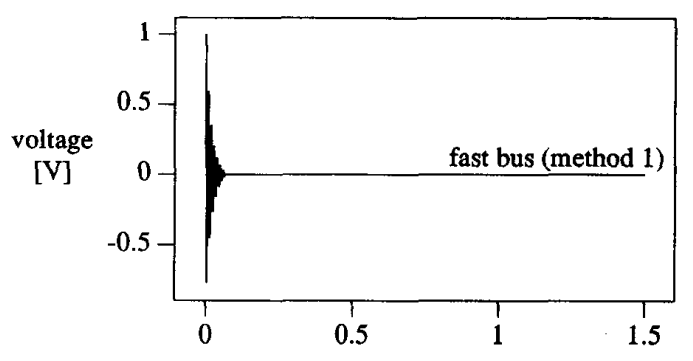

(a)

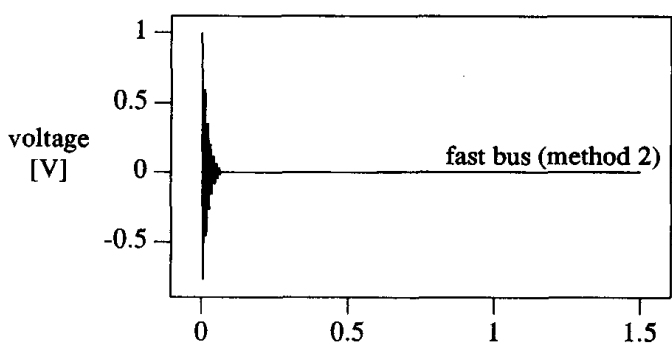

(b)

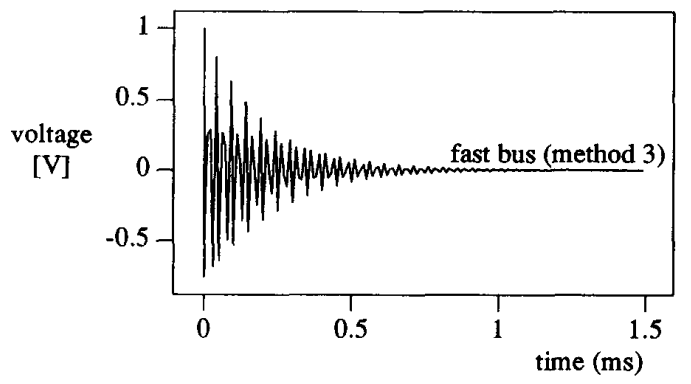

(c)

Figure 5. Voltage at the fast bus using lossy transmission lines

From Figures 5 we can see that method 2 predicts accurately the voltage at the fast bus, while method 3 gives very inaccurate results. In Figure 6 we show the details, at a different scale, of the fast transient using methods 1 and 2 . As we can see, the transient voltages obtained from both methods are indistinguishable.

Figure 7 presents the voltages at the source and at the slow bui using all three methods. As expected, method 3 gives good results at the slow bus since the integration step $\mathrm{H}$ is adequate for the slow part. Clearly, a necessary condition for obtaining good results is the frequency dependent modeling of the transmission lines.

In conclusion:

(a) Methods 1 and 2 give equally good results in both fast and slow buses. Method 2 is, however, more efficient than method 1 .

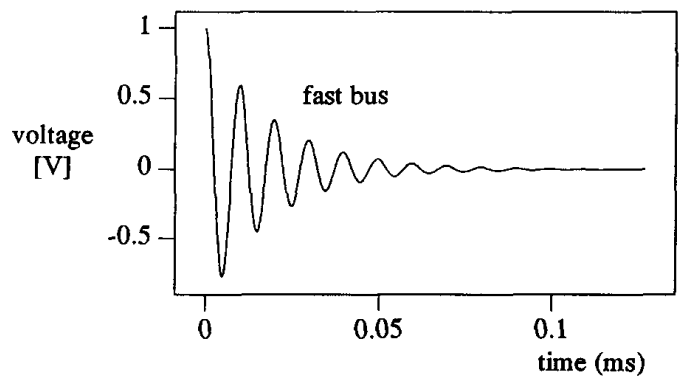

Figure 6. Details of the voltage at the fast bus (methods 1 and 2)

(b) Method 3 gives adequate results for slow buses if the transmission lines are modeled as frequency dependent.

(c) Method 3 works well for all buses when the fast transient is not excited (it could be excited by a steep incident wave or by non-zero initial conditions).

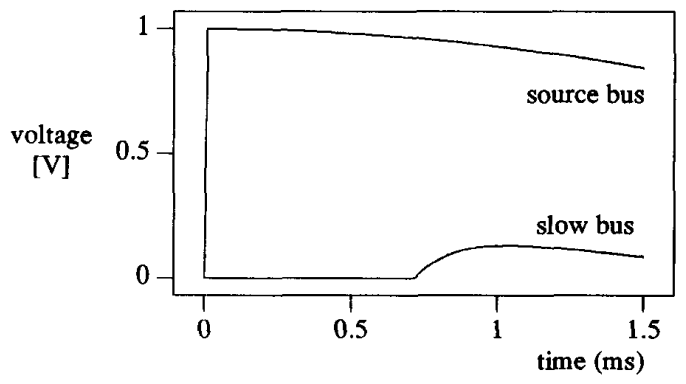

Figure 7. Voltage at the slow buses with methods 1,2 and 3

\subsection{Evaluation of Efficiency and Accuracy}

A bigger system consisting of 10 buses and 14 transmission lines was used for the evaluation of the efficiency and accuracy of the new method. In Figure 8 we show the topology of the system. There are 8 slow loads (S), one fast load (F), and a voltage source. As in the previous example, the transmission lines are slow compared with the fast load. The simulated transient is the energization of bus 10 at $t=0$. As before, the fast transient is excited by an initial charge on the capacitor at node 1 . The integration steps used in the simulation were: $\mathrm{H}=10 \mu \mathrm{s}$ and $\mathrm{h}=$ $0.1 \mu \mathrm{s}$. Figures 9 show the results with voltages at buses near the fast load being displayed.

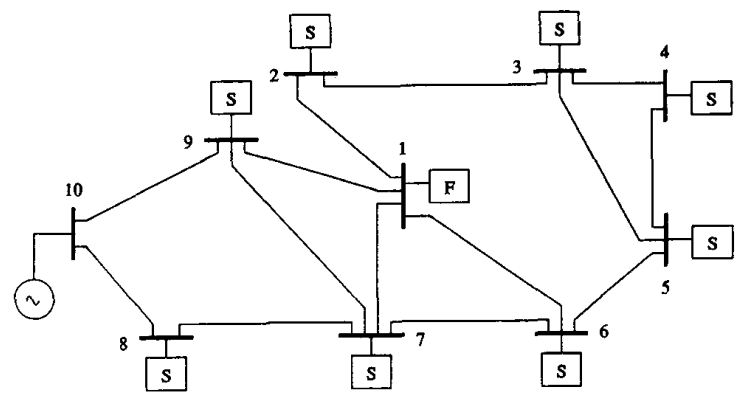



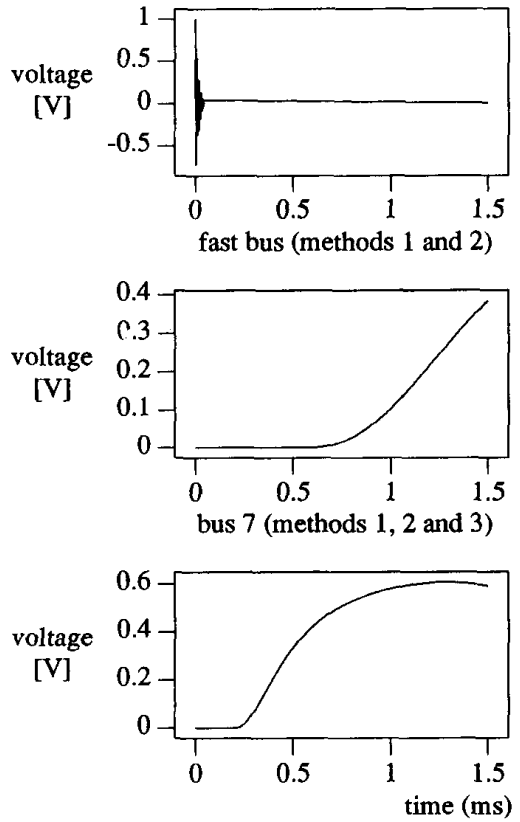

bus 9 (methods 1,2 and 3 )
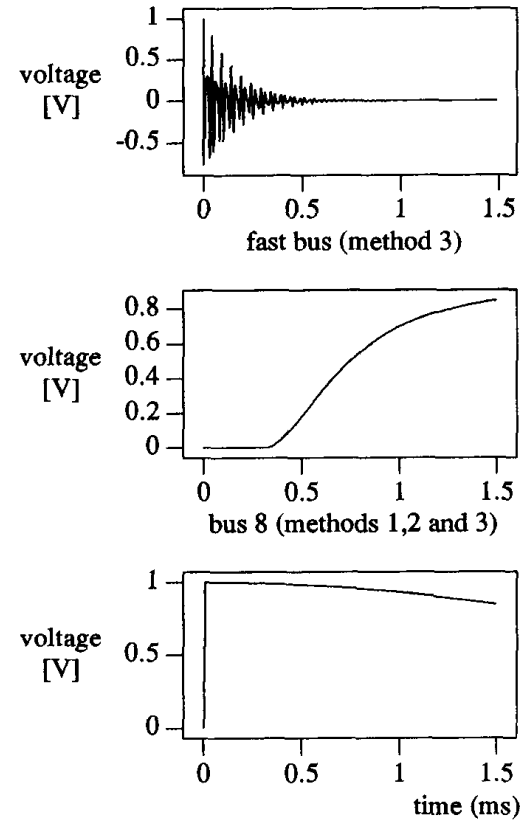

bus 10 (methods 1,2 and 3 )

Figure 9. Transient voltages for the 10 bus system

Although method 3 does not predict accurately the transient voltage at the fast bus, the voltages at the slow buses are computed with precision. Method 2 gives good results for the fast bus as well, at substantial savings of computer time; see Table 1.

\begin{tabular}{|c||c|}
\hline method & cpu time \\
\hline \hline 1 & 18.72 \\
2 & 1.95 \\
3 & 0.18 \\
\hline
\end{tabular}

Table 1. Cpu time for the simulation of the system of Figure 8

From the table we can see that the computer times differ by one order of magnitude in methods 1 and 2 , and similarly, in methods 2 and 3 . A simple explanation is that with method 3 we perform 150 basic calculations (for the $1.5 \mathrm{~ms}$ time interval) on 10 buses, giving a total of 1500 calculations. Method 2 includes 150 calculations on 9 buses and 15000 on one bus, which results in 16350 calculations (slightly above 10 times more). With method 1 we perform 15000 calculations on 10 buses, resulting in a total of 150000 calculations (100 times more than with method 3 and approximately 10 times more than with method 2).

The maximum difference (in the transient voltages) between methods 1 and 2 for the fast bus is less than $0.01 \%$. For the slow buses the maximum differences are smaller than $1 \%$ and the average differences are less than $0.2 \%$. Because of these remarkably small errors, two or three curves cannot be seen from the simulations of Figures 5,6,7 and 9. When comparing methods 1 and 3 we get for the slow buses maximum differences smaller than $5 \%$ and the average differences are less than $1 \%$.

\section{CONCLUSIONS}

The paper describes a methodology for using two or more time steps in the trapezoidal integration for the calculation of transients in a program based on traveling waves, such as the EMTP.
It is shown that the dual time step interface can easily be implemented between a line and a bus by the simple approach of interpolating the incident wave and smoothing the reflected wave. The domain of application is with lines modeled as frequency dependent.

The use of dual (or multiple) time steps can lead to very substantial computational savings, if applied to the right problem, without any loss of accuracy. It is believed that implementation in the EMTP would not be a difficult task.

\section{ACKNOWLEDGEMENTS}

Financial support by the Natural Sciences and Engineering Research Council of Canada is gratefully acknowledged. The second author wishes to express his gratitude to the National Polytechnic Institute of Mexico for the study leave at the University of Toronto.

\section{REFERENCES}

[1] A. Vladimirescu, K. Zhang, A.R. Newton, D.O. Pederson, A. Sangiovanni-Vincentelli, "Spice Version 2G User's Guide", 1981.

[2] A.T. Davis, "ECA-2 Electronic Circuit Analysis", Tatum Labs, Inc., 1989.

[3] F. de León and A. Semlyen, "Efficient Calculation of Elementary Parameters of Transformers", IEEE Transactions on Power Delivery, Vol. 7, No. 1, January 1992, pp. 376-383.

[4] F. de León and A. Semlyen, "Reduced Order Model for Transformer Transients", IEEE Transactions on Power Delivery, Vol. 7, No. 1, January 1992, pp. 361-369.

[5] F. de Leon and A. Semlyen, "Time Domain Modeling of Eddy Current Effects for Transformer Transients", paper no. 92 WM 251-9 PWRD presented at the 1992 IEEE/PES Winter Meeting.

[6] A. Semlyen and A. Dabuleanu, "Fast and Accurate Switching Transient Calculations on Transmission Lines with Ground Return Using Recursive Convolutions", IEEE Transactions on Power Apparatus and Systems, Vol. PAS-94, No. 2, March/April 1975, pp. 561-571. 
[7] J. Marti, "Accurate Modelling of Frequency Dependent Transmission Lines in Electromagnetic Transient Simulations", IEEE Transactions on Power Apparatus and Systems, Vol. PAS101, No. 1, January 1982, pp. 147-157.

\section{APPENDIX \\ Dual Step Size Trapezoidal Simulation in Lumped Parameter Systems}

As mentioned in the Introduction, it is generally not true that individual system components can be characterized in an absolute way as slow or fast. The eigenvectors associated with such modes give an indication of by how much these modes participate in each state variable. It is therefore crucial to the success of the dual time step integration method that there be some truly valid, perhaps physically based, reasons for considering a subsystem to be "slow". This requirement is even more imperative in the presence of nonlinear elements. Moreover, it is equally important that the terminal variables, which depend on both the slow and fast connected components, should also be smooth. In what follows, we adopt these assumptions of slow dynamic behavior for the slow subsystem and the interface variables. We describe them by the state equations

$$
\begin{gathered}
\dot{x}=A x+B u+E e \\
y=C x+D u
\end{gathered}
$$

Here, $e$ denotes sources internal to the slow subsystem, while $u$ (voltage) and $y$ (current) are interface variables to the "fast" external subsystem to be connected to it. Both subsystems will end up being represented by their Norton equivalents. To obtain this for the slow subsystem, we discretize (A-1) using the trapezoidal rule starting from initial values marked by subscript 0 . It yields

$$
\left(I-\frac{A \Delta t}{2}\right) x=\left(I+\frac{A \Delta t}{2}\right) x_{0}+\frac{B \Delta t}{2}\left(u+u_{0}\right)+\frac{E \Delta t}{2}\left(e+e_{0}\right)
$$

Based on the assumption of slowness we expect that all eigenvalues of $A \Delta T / 2$ are $\ll 1$, so that

$$
\left(I-\frac{A \Delta t}{2}\right)^{-1}=I+\frac{A \Delta t}{2}+O\left((\Delta t)^{2}\right)
$$

Therefore, (A-3) yields, neglecting terms in $(\Delta t)^{2}$,

$$
x=(I+A \Delta t) x_{0}+\frac{B \Delta t}{2}\left(u+u_{0}\right)+\frac{E \Delta t}{2}\left(e+e_{0}\right)
$$

Substitution of (A-5) into (A-2) gives

$$
\begin{aligned}
& y=G u+y_{\text {hist }} \\
& \text { where } \\
& G=D+\frac{C B}{2} \Delta t
\end{aligned}
$$

and

$$
y_{\text {hist }}=C x_{0}+C\left(A x_{0}+\frac{B}{2} u_{0}+E \frac{e+e_{0}}{2}\right) \Delta t
$$

We have thus obtained the linear equation (A-6), in the terminal variables $y$ and $u$, which can be viewed as a Norton equivalent of the slow subsystem. Its parameters, however, $G$ and $y_{\text {hist }}$ of (A-7) are not constant but linear functions of $\Delta t(h \leq \Delta t \leq H)$. This means that we have a variable Norton equivalent which can be interfaced with the fast external subsystem as it evolves at small steps $h$ within the larger time step $H$ of the slow subsystem. During its evolution we simply set in (A7) $\Delta t=k h$, for the $k$ th step in the fast subsystem.

The saving in the approach described above results from the fact that the state variables in the slow subsystem do not have to be updated for each of the many small steps $h$.

To illustrate the use of the dual step size method consider the circuit shown in Figure A-1. We have performed simulations using three methods:

(1) Small step size for the entire circuit: $h=0.1 \mu$ s (standard procedure).

(2) Dual step size: $\mathrm{H}=5 \mu$ s for the slow part and $\mathrm{h}=0.1 \mu$ s for the fast part, ratio $\mathrm{H} / \mathbf{h}=\mathbf{5 0}$.

(3) Large step size for the whole system: $\mathrm{H}=5 \mu \mathrm{s}$.

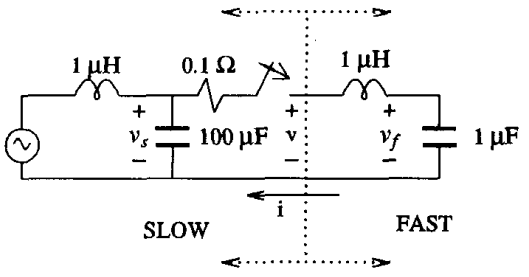

Figure A-1. Circuit for the simulation of dual time step

In Figure A-2 we show the results of the simulation. Each plot in the figure presents the voltages of the capacitors. $v_{f}$ is the voltage of the "fast" capacitor ( $1 \mu \mathrm{F}$ - on the right hand side of the circuit). $v_{s}$ is the voltage of the "slow" capacitor $(100 \mu \mathrm{F}$ - on the left hand side of the circuit). As we can see from the figure, methods 1 and 2 give similar results while method 3 predicts wrongly the voltage on the fast side of the circuit.
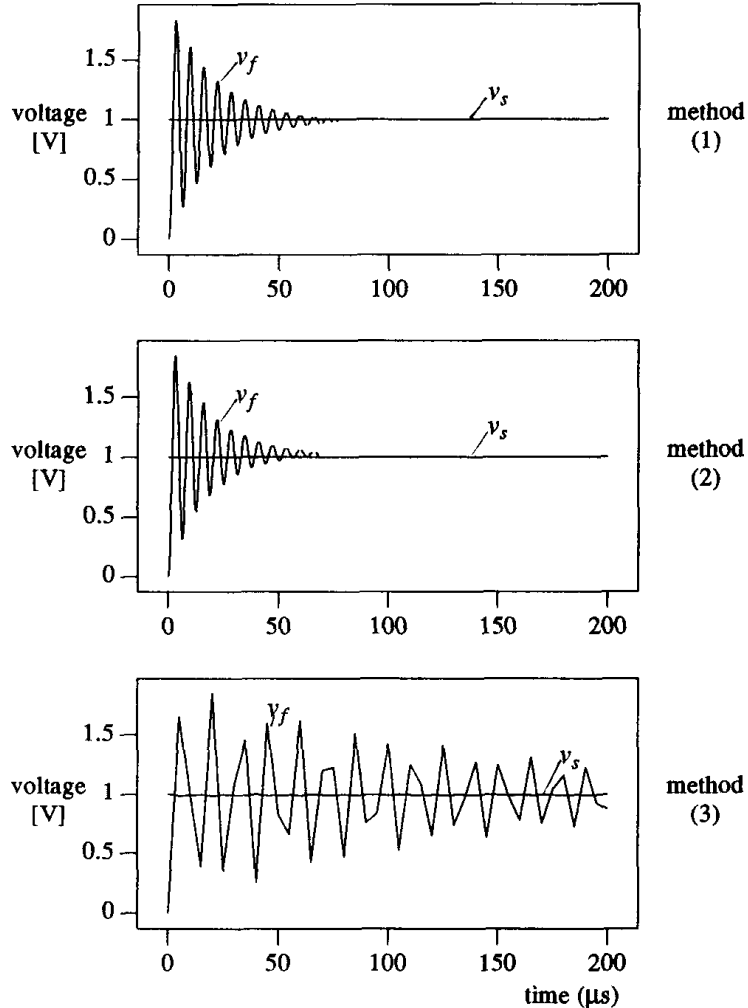

Figure A-2. Simulation of the dual step size

We strongly emphasize that the dual time step method described in this appendix is not applicable for a general lumped parameter network. Its applicability is restricted to circuits with virtually decoupled eigenvalues and slow changing interface variables. Our example meets both conditions: the eigenvalues are almost independent and the interface variables (voltage $v$ and current $i$ in Figure A-1) vary at the speed of the slow system. 
Adam Semlyen ( $\mathrm{F}^{\prime} 88$ ) was born and educated in Rumania where he obtained a Dipl. Ing. degree and his Ph.D. He started his career with an electric power utility and held an academic position at the Polytechnic Institute of Timisoara, Rumania. In 1969 he joined the University of Toronto where he is a professor in the Department of Electrical Engineering. His research interests include the steady state and dynamic analysis of power systems, electromagnetic transients, and power system optimization.
Francisco de León was born in Mexico, in 1959. He received his B.Sc. degree and his M.Sc. degree (summa cum laude) from the National Polytechnic Institute of Mexico, in 1983 and 1986, respectively. From 1984 to 1987 he worked as a lecturer at the same institute. He has obtained a Ph.D. degree in 1992, on a topic of transformer modeling, at the University of Toronto where he currently continues his research as a postdoctoral fellow. His main research interests include electromagnetic fields and transients. 


\section{Discussion}

Huang Wei-Gang ( Department of Electrical Engineering, Tsing-hua University, Beijing, P. R. China) The discusser wish to compliment the authors for their very interesting paper on using dual or multiple time steps for computation of electro-magnetic transients. The selection of the time step used in the computation of electo-magnetic transients usual$1 y$ is dependent on the slope of the applied waveform, the travel time of the shortest line stub or the highest frequency of the components in the system. For example, if the detailed section-to-section or turn-to-turn voltage gradients of a transformer operating in a substation experienced a incoming lightning surge or the lightning surge stresses across insulation paths of a tower being struck by lightning are expected to be calculated, the transfomer winding has to be devided into small sections and the tower has to be simulated by many short line stubs [1] [2]. These sections or line stubs correspond to fast components which determine the size of the time step, and the rest of the lines or elements might be slow. If the method of multiple time steps presented in the paper can be used in the calculation it will lead to substantial computational saving.

There are a few points the discusser would like to make clear.

Can we make following statments that the fast transient oscillations appearing at the fast bus and at the slow bus in a lossless system theoretically exist; it is only in the sense that in reality transmission lines are lossy, so, the oscillations at the slow bus and those at the fast bus reflected from the source bus should be viewed as non-physical; the multiple time steps methodology can not be used in the lossless systems, since fast transient oscillations on the lossless 1 ines will be distored by the "smoothing " procedure?

It seems that Fig. 3 and Fig. 4 (b) have not been chosen properly. According to Fig $4(\mathrm{~b})$ and Fig 7 , the transient appearing at slow bus is at $0.7 \mathrm{~ms}$. It shows that the distance from bus 3 to bus 2 is 210 km. The statment in the paragraph below Fig. 3 ".... fast transient oscillations appearing at the slow bus at $0.6 \mathrm{~ms} . . . . "$ is not correct and it also does not coincide with Fig. 3. The discusser considers that the fast transient oscillations appearing at slow bus 2 should not at $0.6 \mathrm{~ms}$ but at $1.1 \mathrm{~ms}$ ( the travel time from bus 3 via bus 1 to bus 2 is equal to $0.5 \mathrm{~ms}$ plus $0.6 \mathrm{~ms}$ )( see Fig. A).

The authors' comments to the above mentioned points will be appreciated.

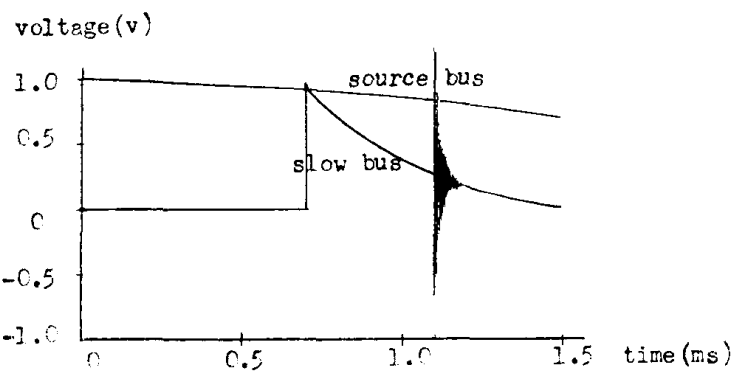

Fig. A voltage at source bus and slow bus

[1] Huang Wei-Gang and Bai Jin-Xia: Surges in substation and Transformer Windings under Operating conditions. High Voltage Engineering, No.3, 1990, pp $9-14$.

[2] Huang Wei-Gang: Calculation of the Lightning performance of a $220 \mathrm{kV}$ Compact line. Proceedings of International Conference on Power System Technology, Beijing, China, 1991, Vol.2, pp 742-747.
ALI ABUR \& HARI SINGH, Texas A \& M University, College Station, Texas; We congratulate the authors for their interesting paper. The method presented is most useful when the study system contains identifiable fast, medium and slow subsystems which are to be solved together to compute the system transients. In such cases, the conventional single time step solution for the whole study system will require the use of a small time step chosen in accordance with the fastest component in the system.

In the case of non-linear elements, a similar restriction on the time step is imposed to ensure that the so-called "knee points" on the piecewise linear characteristics are not crossed abruptly during the simulations. Can such non-linear elements be isolated and solved using a smaller time step than the one used in the rest of the system based on the proposed method in the paper? If so, would the interpolation and smoothing procedures described in sections 2.2.1. and 2.2.2 respectively still remain robust?

The authors' comments on the above will be greatly appreciated.

Manuscript received August 10, 1992.

Adam Semlyen and Francisco de León: We would like to express our appreciation to the discussers for their interest in our paper and for their remarks and questions.

We thank professor Huang for pointing out an error in Figure 3. The labels of $180 \mathrm{~km}$ and $210 \mathrm{~km}$ for two of the lines have been interchanged. Figure $3 \mathrm{a}$ (below) shows the corrected layout. The simulations are otherwise correct. The voltage oscillations appearing at the slow bus at $t=0.6 \mathrm{~ms}$ (traveling time from the fast to the slow bus) are the result of the initial charge of the capacitor at the fast bus, given in order to excite the fast oscillations of Figures 4 and 5.

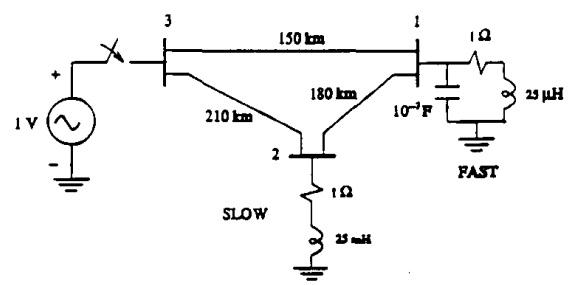

Figure 3a. Corrected version of Figure 3

Professor Huang is correct in his remark that dual or multiple time steps cannot be used if the lines have insufficient damping to confine to the fast buses any local, fast oscillations. If lines with poor damping exist, then all buses connected by such lines (to a fast bus) should be treated as a cluster of fast buses.

Dr. Abur and Mr. Singh are right that the multiple time step methodology could advantageously be applied in the case of nonlinear components with sharp knee points due, perhaps, to the use of piecewise linear characteristics. Interpolation is then still needed since its purpose is to obtain inputs to the fast bus at each small time step $h$. The purpose of smoothing is to make sure that the outgoing wave from the fast bus does not include (the erratic information resulting from) the effect of the fast transient that may exist there at the end of the larger time step $H$ when the information is needed at the other end of the line. Since this will not be the case if the bus with the nonlinear element has no fast dynamics, therefore, smoothing may not be needed for buses with only nonlinear elements.

Manuscript received August 31, 1992. 